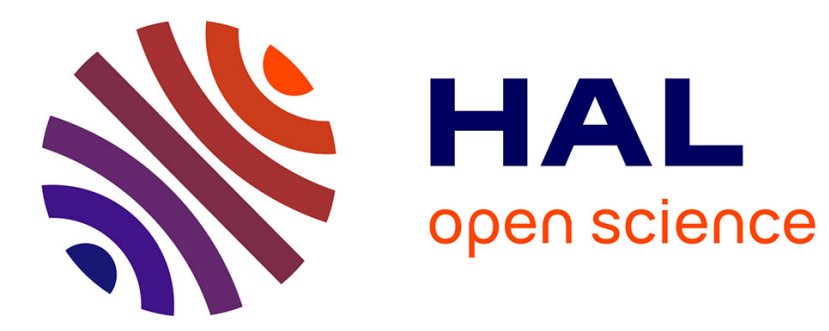

\title{
Contrastes simulés au croisement de défauts d'empilement
}

\author{
André Rocher, Bernard Jouffrey
}

\section{To cite this version:}

André Rocher, Bernard Jouffrey. Contrastes simulés au croisement de défauts d'empilement. Revue de Physique Appliquée, 1969, 4 (4), pp.525-532. 10.1051/rphysap:0196900404052500 . jpa-00243324

\section{HAL Id: jpa-00243324 https://hal.science/jpa-00243324}

Submitted on 1 Jan 1969

HAL is a multi-disciplinary open access archive for the deposit and dissemination of scientific research documents, whether they are published or not. The documents may come from teaching and research institutions in France or abroad, or from public or private research centers.
L'archive ouverte pluridisciplinaire HAL, est destinée au dépôt et à la diffusion de documents scientifiques de niveau recherche, publiés ou non, émanant des établissements d'enseignement et de recherche français ou étrangers, des laboratoires publics ou privés. 


\title{
GONTRASTES SIMULÉS AU GROISEMENT DE DÉFAUTS D’EMPILEMENT
}

\author{
Par André ROCHER et Bernard JOUFFREY, \\ Laboratoire de Physique des Solides, associé au C.N.R.S., Faculté des Sciences d'Orsay, Essonne.
}

(Reçu le 4 septembre 1969.)

\begin{abstract}
Résumé. - Les croisements de défauts d'empilement dans du cobalt-chrome sont étudiés par comparaison de micrographies obtenues par microscopie électronique et de simulations réalisées sur ordinateur. La technique de simulation est analogue à celle donnée par Head. Les calculs des intensités transmise et diffractée sont faits à partir de la théorie dynamique à deux ondes dans un cristal parfait. La correspondance simulation-micrographie est satisfaisante.
\end{abstract}

Abstract. - The image contrast at intersecting stacking faults is studied by electron microscopy and computer simulation in a cobalt-chromium alloy. The technique for simulation of electron micrographs is similar to the one given by Head. The calculations of transmitted and diffracted intensities are carried out with the two beam dynamical theory in a perfect crystal. The agreement between simulated and experimental images is quite reasonable.

La théorie des contrastes en microscopie électronique par transmission est maintenant bien comprise. Elle permet de donner au moins une explication semiquantitative à de nombreux résultats expérimentaux au moyen de courbes donnant des profils d'intensités. Avec l'amélioration des performances des ordinateurs, il est apparu possible de simuler directement des micrographies électroniques traduisant la présence de défauts cristallins. Il suffit pour cela de dresser des cartes d'intensités donnant les probabilités de passage des électrons sur la face de sortie de l'échantillon étudié. L'avantage d'une telle méthode est de permettre une comparaison directe de l'expérience et du résultat théorique.

Tout d'abord, nous rappellerons brièvement la formulation de la théorie dynamique que nous utilisons. Nous donnerons ensuite quelques indications sur la programmation des simulations de croisement de défauts d'empilement et nous conclurons en comparant sur quelques cas précis les résultats théoriques et expérimentaux.

La plupart de ces résultats ont été présentés récemment $[1,2]$. Le traitement ci-dessous peut être trouvé plus détaillé dans la référence [3].

I. Théorie dynamique. - A. Formulation GÉnérale. Approximations. - Le traitement des électrons dans un cristal en microscopie électronique se fait typiquement à l'aide de la théorie des électrons presque libres dans un potentiel périodique [4]. On part de l'équation de Schrödinger :

$$
\frac{\hbar^{2}}{2 m} \Delta \Psi+(E-V) \Psi=0
$$

Puisque l'énergie potentielle $V$ est périodique, la solution de cette équation peut être mise sous forme de la somme d'ondes de Bloch :

$$
\Psi=\sum_{k} u_{k}(r) \mathrm{e}^{i \mathbf{k r}} .
$$

Pour des réflexions de Bragg, cette somme peut se réduire à deux termes. On obtient expérimentalement ce cas particulier pour certains matériaux lorsque l'énergie des électrons n'est pas trop élevée (typiquement $100 \mathrm{keV})$. Il est d'autant plus facile d'être dans cette situation que le cristal utilisé est composé d'atomes de faible masse atomique car l'épaisseur observable est alors importante. Cette situation de deux ondes (une seule onde excitée) nous servira de base de calcul.

Dans ce cas, des approximations propres à la microscopie électronique permettent de simplifier le système d'équations caractérisant ce problème :

- Approximation en colonne. - Les électrons entrant dans le cristal suivant une direction donnée ne s'en écartent pas de façon notable, qu'ils soient transmis ou diffractés. En effet, l'approximation en colonne est justifiée parce que, en microscopie classique (100 keV), les angles de Bragg caractéristiques ont des valeurs de l'ordre du demi-degré et que les épaisseurs traversées ne sont pas trop grandes. Le problème de propagation des électrons dans le cristal se réduit alors à un problème à une dimension. Les probabilités de passage des électrons (intensités transmises et diffractées) sont alors calculées en chaque point de la face de sortie de façon indépendante. Des résultats numériques ont montré que cette approximation était tout à fait justifiée $[5,6]$. 
- Variation lente des fonctions d'ondes. - Celle-ci permet d'obtenir un système simple d'équations différentielles linéaires du premier ordre. Deux autres hypothèses sont utilisées dans l'étude des contrastes.

- Le traitement des défauts est effectué en supposant que les atomes déplacés conservent leur loi de potentiel. - Seul un déphasage est alors introduit par un défaut. Cette approximation est valable pour de faibles déplacements (loin du cœur d'une dislocation) ou pour des atomes qui conservent leur entourage de premiers voisins (défaut d'empilement). Ce traitement ne permet d'introduire les contrastes de facteurs de structure que dans certains cas particuliers. Ce dernier type de contraste peut être traité d'une manière générale en faisant la somme des potentiels dus à chaque atome.

- Potentiel imaginaire. - L'autre approximation nécessaire pour pouvoir rendre compte des contrastes observés consiste à introduire une partie imaginaire dans le potentiel afin de tenir compte de la diffusion des électrons à grand angle.

Les électrons incidents sont dans notre hypothèse de calcul en majorité transmis et diffractés suivant deux directions privilégiées, mais une partie non négligeable est diffusée dans d'autres directions. Ces électrons, qui ne passent pas par le diaphragme de contraste, sont alors perdus pour la formation de l'image, qu'il s'agisse du fond clair ou du fond noir. On parle alors « d'absorption ».

Pour tenir compte facilement de ce fait, on introduit, comme nous venons de le dire, une partie imaginaire dans le potentiel cristallin :

$$
V(r) \rightarrow V(r)+i V^{\prime}(r) .
$$

Ce traitement dû à Molière [7] a reçu une justification proposée par Yoshioka [8].

La périodicité du potentiel imaginaire permet de rendre compte des effets de contraste anomaux (dépendant de l'orientation du cristal).

En effet, la valeur moyenne du potentiel imaginaire $i v_{0}^{\prime}$ n'introduit qu'un facteur d'atténuation commun à toutes les ondes (pour une épaisseur donnée). G'est le terme d'absorption normale (ne dépendant pas de l'orientation du cristal). Par contre, les composantes de Fourier $i v_{g}^{\prime}$ du potentiel imaginaire introduisent une absorption anomale.

B. Formulation de Howie et Whelan [9]. Ces approximations permettent d'écrire un système simple pour un cristal écarté ou non de l'angle de Bragg et contenant une perturbation cristalline :

$$
\begin{aligned}
& \frac{\mathrm{d} \varphi_{0}}{\mathrm{~d} z}=+i \pi\left(-w+i C_{0}\right) \varphi_{0}+i \pi\left(1+i C_{g}\right) \varphi_{g} \mathrm{e}^{i \alpha(z)} \\
& \frac{\mathrm{d} \varphi_{g}}{\mathrm{~d} z}=i \pi\left(1+i C_{g}\right) \varphi_{0} \mathrm{e}^{-i \alpha(z)}+i \pi\left(w+i C_{0}\right) \varphi_{g} .
\end{aligned}
$$

Dans ce système, la distance d'extinction est prise comme unité de longueur; c'est une fonction du maté- riau, de l'énergie des électrons et de la réflexion de Bragg considérée. Les coefficients introduits sont :

$$
w=s \xi_{g}
$$

$s$, écart à l'angle de Bragg; $\xi_{g}$, distance d'extinction caractérisant le cristal et la réflexion;

$$
C_{\mathbf{0}}=\frac{V_{0}^{\prime}}{V_{g}} ; \quad C_{g}=\frac{V_{g}^{\prime}}{V_{g}}
$$

coefficients d'absorption normale et anomale ;

$$
\alpha(z)=2 \pi \mathbf{g} \cdot \mathbf{R}(z)_{x y}
$$

déphasage introduit par un défaut dont le déplacement par rapport au cristal parfait à la cote $z$ et suivant la colonne $(x, y)$ est $\mathbf{R}(z)_{x y}$.

Les calculs de contrastes consistent à calculer $\varphi_{0}$ et $\varphi_{g}$ en chaque point de la face de sortie du cristal.

Le problème dans le cas des défauts d'empilement a une solution simple. En effet, ces défauts plans séparent le cristal en deux parties restant parfaites ( $\alpha=$ Cte pour la partie déplacée). Le traitement des contrastes sera alors simplement une étude de propagation des électrons dans deux cristaux parfaits, les conditions de passage de l'un à l'autre étant à préciser.

Le système dans un cristal parfait est à coefficients constants et a pour solution des sommes d'exponentielles qui s'écrivent sous la forme :

$$
\begin{aligned}
& \varphi_{0}=A \exp \pi \gamma_{1} z+B \exp \pi \gamma_{2} z \\
& \varphi_{g}=\beta_{1} A \exp \pi \gamma_{1} z+\beta_{2} B \exp \pi \gamma_{2} z
\end{aligned}
$$

avec :

$$
\begin{gathered}
q \#\left(1+w^{2}\right)^{1 / 2} \\
\gamma_{1}=-C_{0}-\frac{C_{g}}{q}+i q ; \quad \gamma_{2}=-C_{0}+\frac{C_{g}}{q}-i q \\
\beta_{1}=\frac{i(w+q)-C_{g} / q}{i\left(1+i C_{g}\right)} ; \quad \beta_{2}=\frac{i(w-q)+C_{g} / q}{i\left(1+i C_{g}\right)}
\end{gathered}
$$

pour $w=0$, on vérifie que $\beta_{1}=-\beta_{2}=1$.

C. Formulation matricielle [10]. - a) Cristal parfait. - Le système de solutions peut être mis sous forme matricielle :

$$
\begin{array}{r}
\left(\begin{array}{l}
\varphi_{0} \\
\varphi_{g}
\end{array}(z)\right)=\left(\begin{array}{cc}
1 & 1 \\
\beta_{1} & \beta_{2}
\end{array}\right)\left(\begin{array}{rr}
\exp \pi \gamma_{1} z & 0 \\
0 & \exp \pi \gamma_{2} z
\end{array}\right)\left(\begin{array}{l}
A \\
B
\end{array}\right) \\
=\beta \Gamma(z)\left(\begin{array}{l}
A \\
B
\end{array}\right)
\end{array}
$$

$A$ et $B$ sont déterminées grâce aux conditions initiales en $z=0$ par la relation :

$$
\left(\begin{array}{l}
A \\
B
\end{array}\right)=\beta^{-1}\left(\begin{array}{l}
\varphi_{0} \\
\varphi_{g}
\end{array}(0)\right) .
$$


Nous pouvons alors relier les ondes incidentes et les ondes sortant du cristal :

$\left(\begin{array}{l}\varphi_{0} \\ \varphi_{g}\end{array}(z)\right)=\beta \Gamma(z) \beta^{-1}\left(\begin{array}{l}\varphi_{0} \\ \varphi_{g}\end{array}\right)=M(z)\left(\begin{array}{l}\varphi_{0} \\ \varphi_{g}\end{array}(0)\right)$

$M(z)$ sera la matrice de « propagation » dans le cristal parfait. Ces solutions sont simples, notamment dans le cas où l'écart à l'angle de Bragg est nul. Il est possible de trouver les amplitudes en fonction de l'épaisseur par une construction géométrique simple (voir annexe).

b) Cristal contenant un ou plusieurs défauts d'empilement $[11,12]$. - Nous avons dit plus haut qu'un défaut d'empilement introduit sur le potentiel un déphasage constant dans la partie déplacée. Un changement de fonction $\varphi_{g} \rightarrow \varphi_{g} \mathrm{e}^{i \alpha}$ permet de retrouver le système du cristal parfait. Dans la formulation matricielle, l'introduction d'un défaut d'empilement sera faite par :

$$
\left(\begin{array}{l}
\varphi_{0} \\
\varphi_{g} \mathrm{e}^{i \alpha}
\end{array}\right)=Q\left(\begin{array}{l}
\varphi_{0} \\
\varphi_{g}
\end{array}\right) \quad \text { avec } \quad Q=\left(\begin{array}{ll}
1 & 0 \\
0 & \mathrm{e}^{i \alpha}
\end{array}\right)
$$

La relation permettant de calculer les amplitudes des fonctions de probabilités dans un cristal contenant un défaut d'empilement sera :

$$
\left(\begin{array}{l}
\varphi_{0} \\
\varphi_{g}
\end{array}\left(z_{1}+z_{2}\right)\right)=Q^{-1} M\left(z_{2}\right) Q M\left(z_{1}\right)\left(\begin{array}{l}
\varphi_{0} \\
\varphi_{g}
\end{array}(0)\right) .
$$

Cette relation analogue à celle donnée par Gevers et al. [11] permet de traiter le contraste pour un cristal contenant un ou plusieurs défauts d'empilement.

II. Mise en place du calcul. Programmation. Deux sortes de problèmes se posaient pour réaliser ces simulations :

a) LA DÉTERMINATION DES PARAMÈTRES GARAGTÉRISANT LES GROISEMENTS DE DÉFAUTS D'EMPILEMENT.

— L'écart à l'angle de Bragg est connu à l'aide des lignes de Kikuchi visibles sur les diagrammes de diffraction.

- Le coefficient d'absorption normale $C_{0}$ n'intervient pratiquement que dans un facteur multiplicatif commun à toutes les intensités. Notre méthode de simulation permet de le négliger.

- Le coefficient d'absorption anomale $C_{g}$ est un des paramètres à ajuster par approximations successives.

- Le repérage analytique des faces d'entrée et de sortie des électrons dans le cristal ainsi que des plans des défauts d'empilement est également à définir.

Pour déterminer les équations des plans de défauts d'empilement qui se croisent, nous faisons l'hypothèse simplificatrice que le plan de la face de sortie du cristal est normal au faisceau électronique et est parallèle à la plaque photographique. Ceci permet de

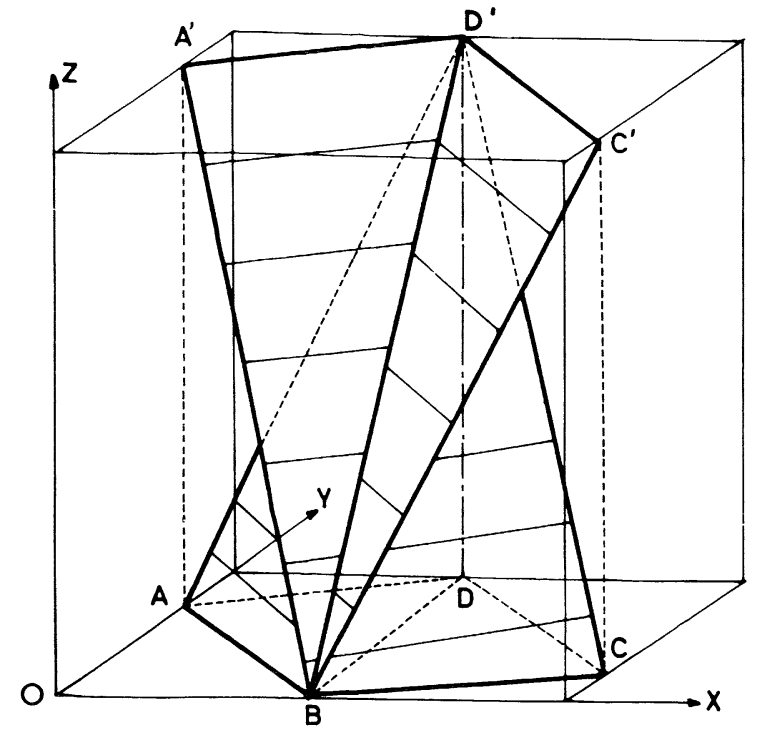

Fig. 1. - Trièdre de référence servant à repérer analytiquement les défauts d'empilement constituant le croisement.

prendre un trièdre de référence simple (fig. 1). Le plan $(\mathrm{X}, \mathrm{Y}, \mathrm{O})$ est le plan de la face de sortie, l'axe $Z$ est l'axe de propagation des électrons. Les quatre points $\mathrm{ABCD}$ ( $f$ ig. 2$)$ de coordonnées $\left(X_{\mathrm{I}}, Y_{\mathrm{I}}, O\right)$ et $\left(X_{\mathrm{D}}, Y_{\mathrm{D}}, Z_{\mathrm{D}^{\prime}}\right)$ permettent de déterminer les équations

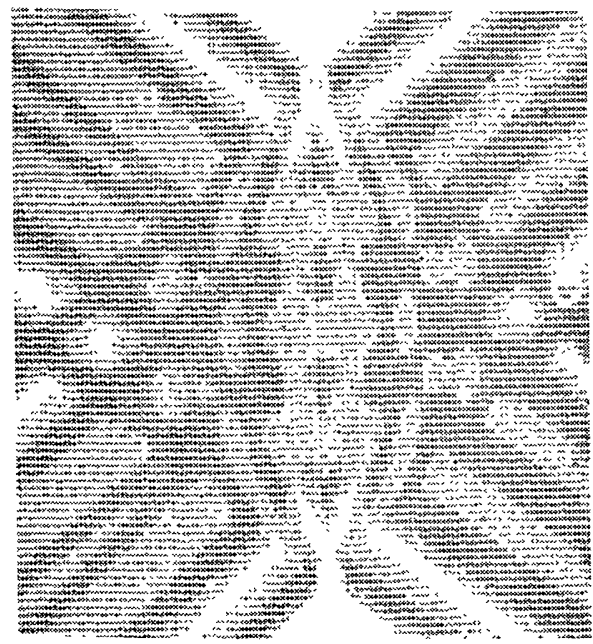

FIG. 2. - Plan de base et plan de l'image.

des plans respectifs $\left(\mathrm{ABD}^{\prime}\right),\left(\mathrm{BCD}^{\prime}\right)$ des deux défauts. De plus, pour que le quadrilatère $\mathrm{ABCD}$ limite effectivement la partie contrastée due au croisement, il est nécessaire que $\mathrm{A}^{\prime}$ et $\mathrm{C}^{\prime}$ soient sur les plans de défaut et sur la face d'entrée; ( $\left(\mathrm{A}^{\prime} \mathrm{D}^{\prime} \mathrm{C}^{\prime}\right)$ permettent alors de déterminer l'équation de la face d'entrée. Les coordonnées $X_{I}$ et $Y_{I}$ n'intervenant que par leurs 
rapports, les unités de définitions sont arbitraires. L'épaisseur $Z_{\mathrm{D}}$ en distance d'extinction est le seul paramètre géométrique du croisement. Elle est déterminée par approximations successives. La géométrie du croisement du défaut est alors connue.

b) Présentation des résultats. Programmation. - La programmation en langage Fortran du calcul des intensités transmise et diffractée ne présente pas de problème bien particulier. Seule la sortie des résultats demandait quelques adaptations. Nous avons utilisé pour la sortie une méthode analogue à celle donnée par Head [13]

Après avoir calculé les intensités transmises et diffractées, nous les comparons aux intensités maximales et associons à chacune d'elles un caractère plus ou moins noircissant pour tenir compte de leur valeur. En imprimant les caractères ainsi obtenus, la simulation est donnée directement par l'ordinateur. Cette méthode a l'avantage de ne demander aucun travail de dépouillement pour comparer expérience et résultats théoriques.

Le programme est utilisé sur l'ordinateur Univac 1108 du centre de calcul de la Faculté des Sciences d'Orsay. Le temps de calcul nécessaire pour effectuer une simulation donnant les fonds clair et noir est de 25 secondes pour 2 fois 12500 points.

III. Résultats. - Cette étude a été faite pour étudier des contrastes au croisement de défauts d'empilement dans du cobalt-chrome, observés par Jouffrey et al. [14].

a) Nous avons étudié des croisements de deux sortes. Les défauts d'empilement dans le premier cas introduisent sur les ondes des déphasages de même signe et de signe opposé dans le second cas.

1) Les déphasages introduits par les deux défauts sont les mêmes, $\alpha=-\frac{2 \pi}{3}$.

Il faut remarquer sur la micrographie que :

- L'intersection se comporte comme un défaut irıtroduisant un déphasage égal à la somme des déphasages des défauts se croisant, c'est-à-dire comme un défaut introduisant un déphasage $\alpha=+\frac{2 \pi}{3}$. En fond clair, les extrémités de l'intersection sont blanches (fig. 3 a et b).

- Le centre du croisement est caractérisé par un système de flèches orientées vers la face (entrée, sortie) la plus proche. En fond clair, la symétrie du système est vérifiée.

FIG. 3 a. - Micrographie $(100 \mathrm{keV})$ de croisement de défauts d'empilement introduisant le même déphasage $\alpha=-\frac{2 \pi}{3}: w=0$.

b) Simulation du fond clair correspondant.

c) Simulation du fond noir.

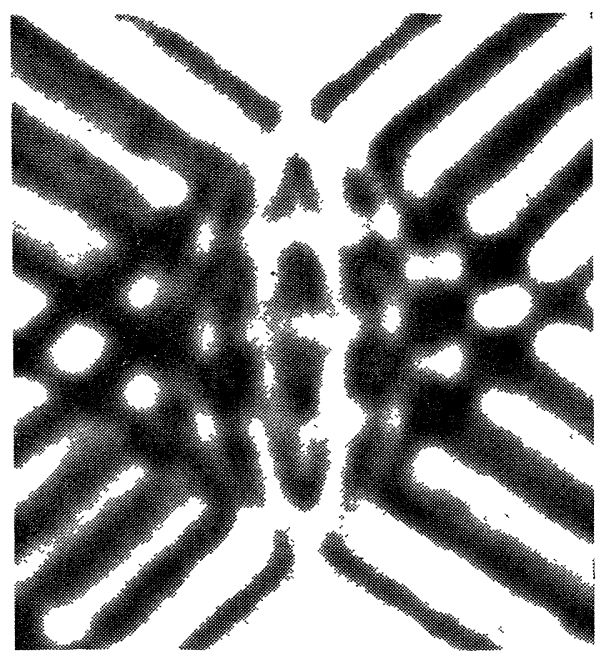

a

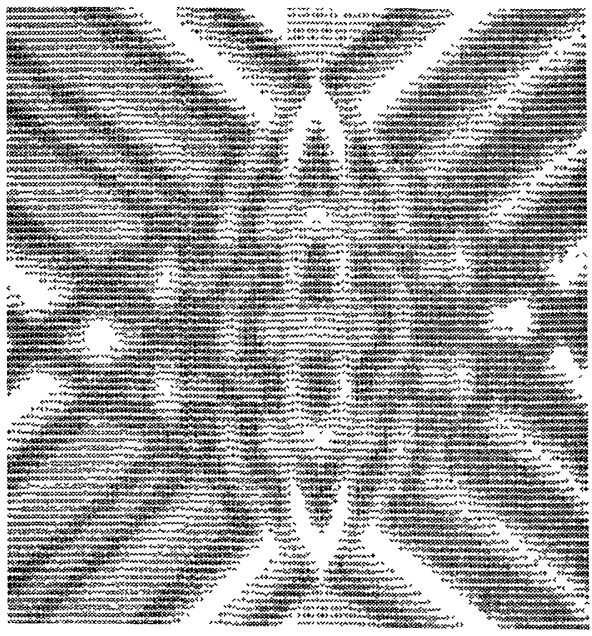

b

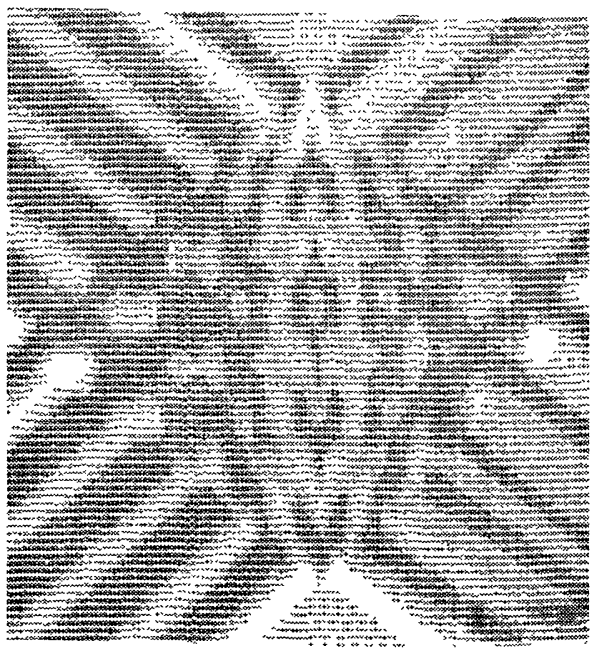

c 
La simulation correspondante rend compte de façon satisfaisante des contrastes observés. Pour l'obtenir, nous avons dû fixer l'absorption anomale par $\xi_{g}^{\prime}=12,5 \xi_{g}$ qui correspond à $C_{g}=0,08$. L'épaisseur au point $\mathrm{D}^{\prime}$ a été déterminée par approximations successives et trouvée égale à $4,7 \xi_{g}$.

En fond noir, l'effet d'absorption anomale introduit une forte dissymétrie. La flèche blanche indique la face d'entrée, la flèche noire complémentaire de la flèche blanche du fond clair indique par quel côté sortent les électrons ( fig. $3 \mathrm{c}$ ).

2) Sur la figure 4, les deux défauts intrinsèques introduisent des déphasages de signes opposés. La somme des déphasages sur l'intersection est nulle : l'intensité observée au centre est celle du fond continu. La simulation a été obtenue pour une épaisseur voisine de $7 \xi_{g}$.

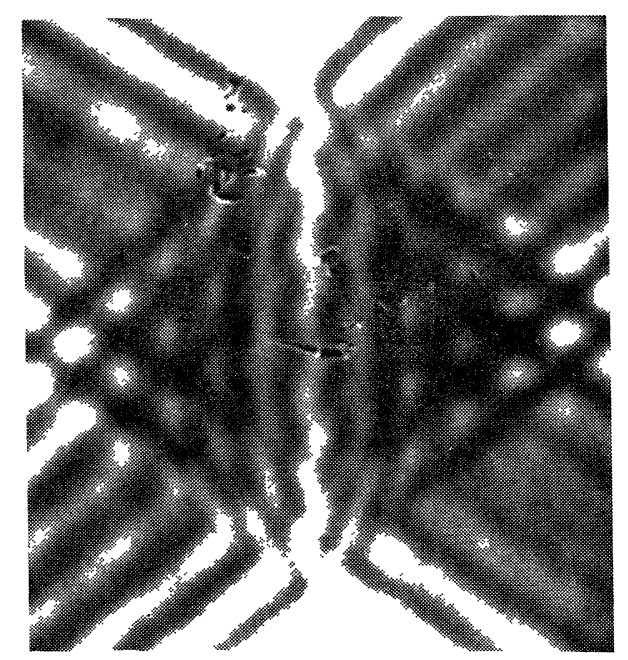

a

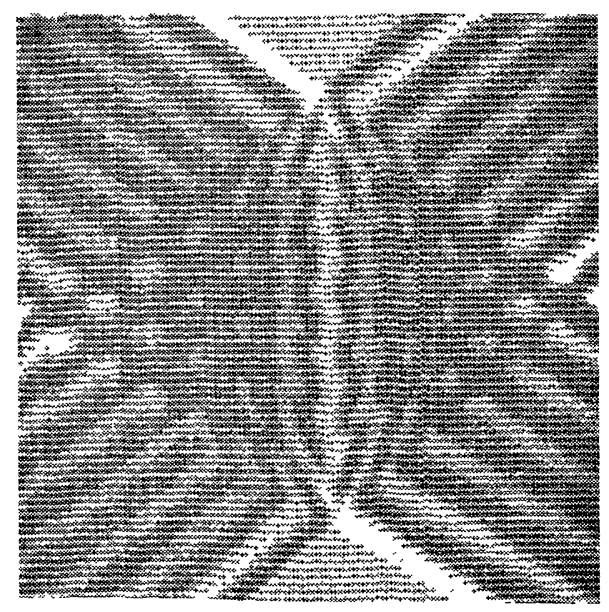

$\mathrm{b}$
Suivant le signe relatif des déphasages, nous pouvons donc avoir des contrastes très différents : le premier avait sur l'intersection un contraste maximum, alors que celui du second était nul, l'intersection ayant l'intensité du fond continu.

b) Étude d'un croisement de défaut d'empilement pour différents écarts à l'angle de Bragg (fig. 5). - Les défauts d'empilement composant le croisement étudié ( fig. 5 a à $5 \mathrm{f}$ ) introduisent tous deux un déphasage $\alpha=-\frac{2 \pi}{3}$.

Les deux micrographies présentées ( $f g .5$ a à $5 \mathrm{~d}$ ) pour des écarts à l'angle de Bragg nul ou très faiblement négatif $(w=-0,15)$ sont assez peu différentes. Pour $w$ assez négatif, l'intensité des franges blanches les plus extérieures est nettement supérieure à celle du fond continu.

Par contre, pour un écart à l'angle de Bragg important avec $w$ positif $(w=1,25)$, la micrographie change d'aspect (fig. 5 e à $5 \mathrm{f}$ ) :

- Le contraste devient plus faible.

- Un système de franges parallèles à l'intersection des deux défauts tend à apparaître. Gevers et al. [12] trouvaient le même effet lorsque les déphasages se retranchaient.

c) Simulation d'une micrographie de croisement de défauts d'empilement prise avec le microscope de Toulouse fonctionnant sous une tension d'un million de volts (fig. 6). - L'essai de simulation que nous avons fait montre, ainsi qu'il est connu, l'insuffisance de la théorie dynamique à deux ondes pour expliquer les contrastes des micrographies obtenues à très haute tension. Cependant, nous avons essayé par simulation d'approcher le plus possible du contraste des micrographies. Le minimum de différence a été obtenu en introduisant dans nos

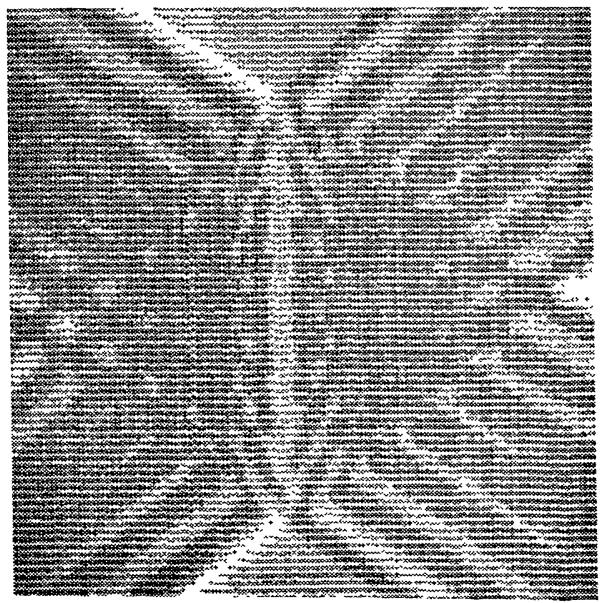

c

FIG. 4 a. - Micrographie $(100 \mathrm{keV})$ de croisement de défauts d'empilement introduisant des déphasages opposés : $w=0$; b) Simulation du fond clair correspondant; c) Simulation du fond noir. 


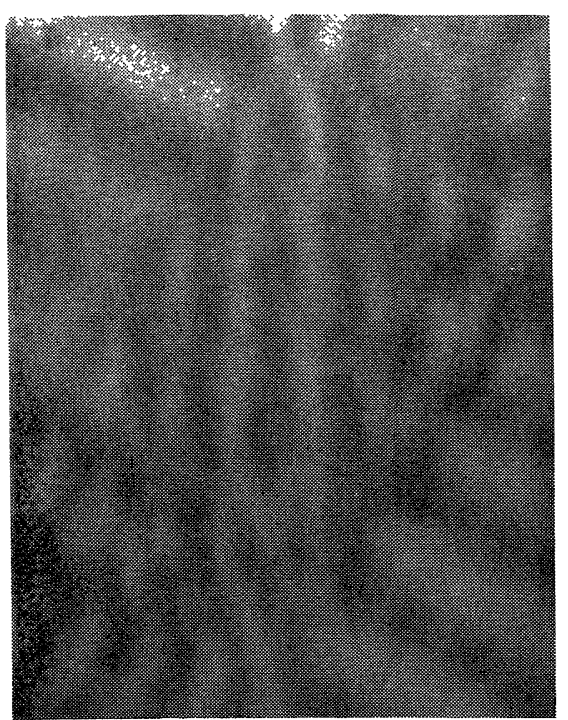

a

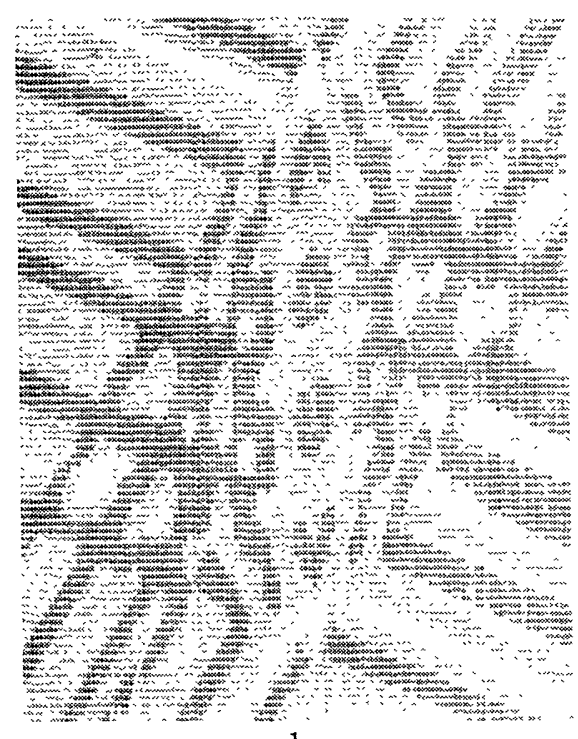

$\mathrm{b}$

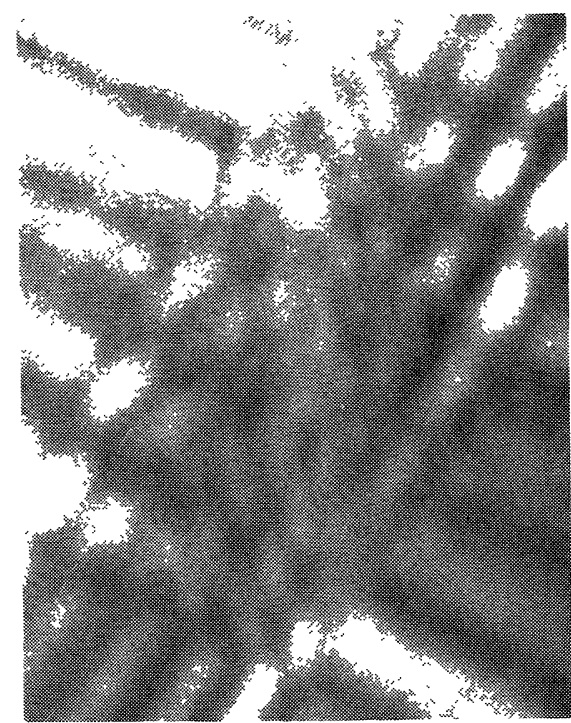

c

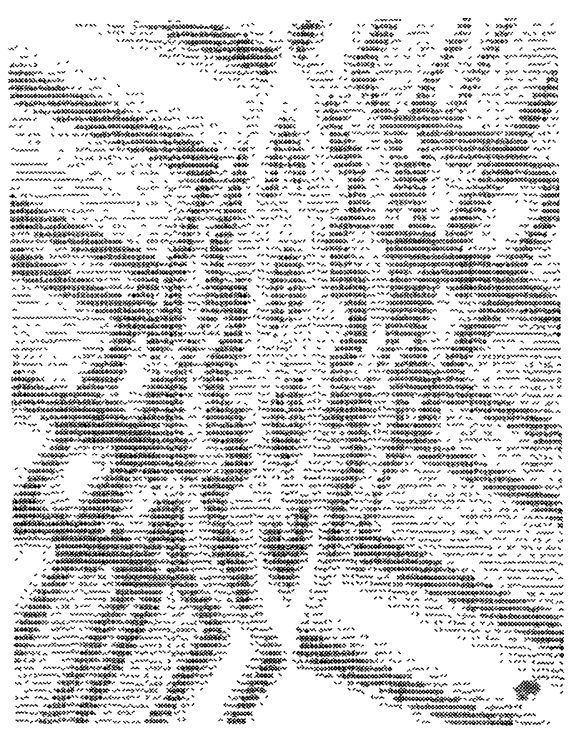

d

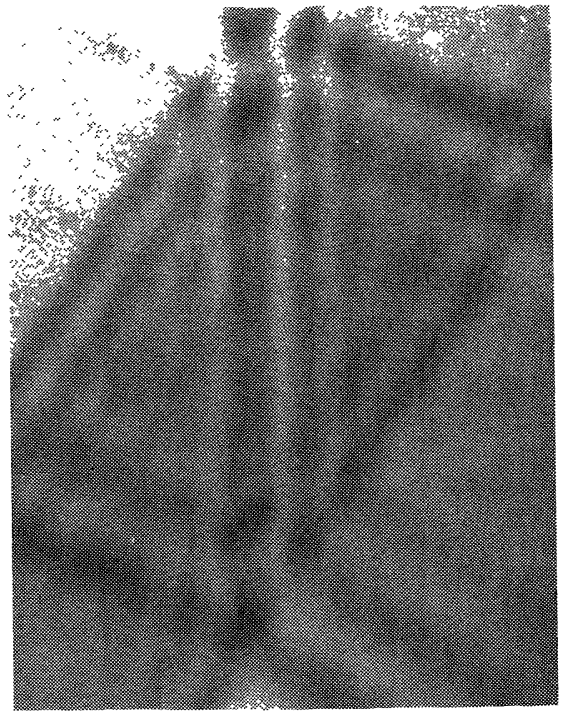

$\mathrm{e}$

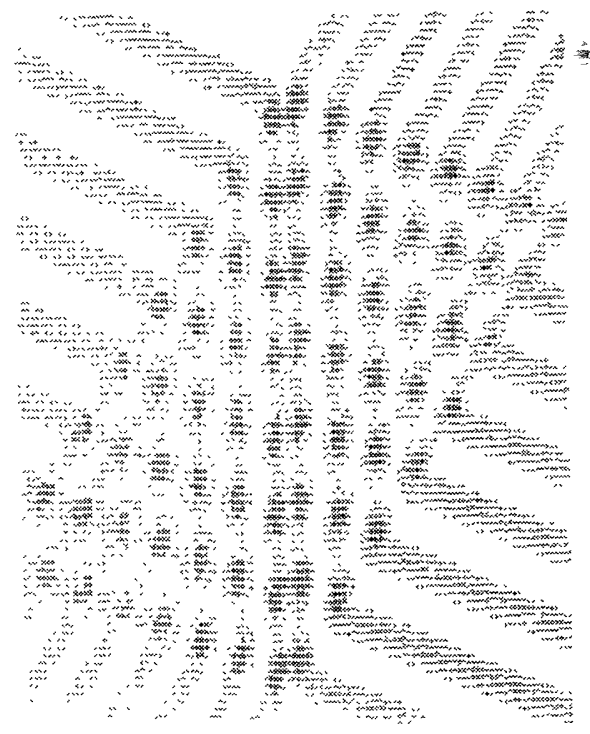

f

Fig. 5. - Micrographie (100 keV) de croisement de défauts d'empilement introduisant le même déphasage $\alpha=-\frac{2 \pi}{3}:$
a) $w=0$.
b) Simulation du fond clair correspondant.
c) $w=-0,15$.
d) Simulation du fond clair correspondant.
e) $w=1,25$
f) Simulation du fond clair correspondant.

équations une absorption anomale $2, \bar{\partial}$ fois plus faible pour des électrons d'une énergie de $1 \mathrm{MeV}$ que pour des électrons de $100 \mathrm{keV}$. Ceci semble en bon accord avec les résultats de Ayrolles [15].

$\mathrm{Du}$ point de vue du contraste, les extrema ont des positions à peu près concordantes sur le cliché et la carte d'intensité. Le dédoublement des franges est obtenu (faible absorption anomale), mais tous les contours sont plus larges et moins contrastés sur la simulation que sur la micrographie. 

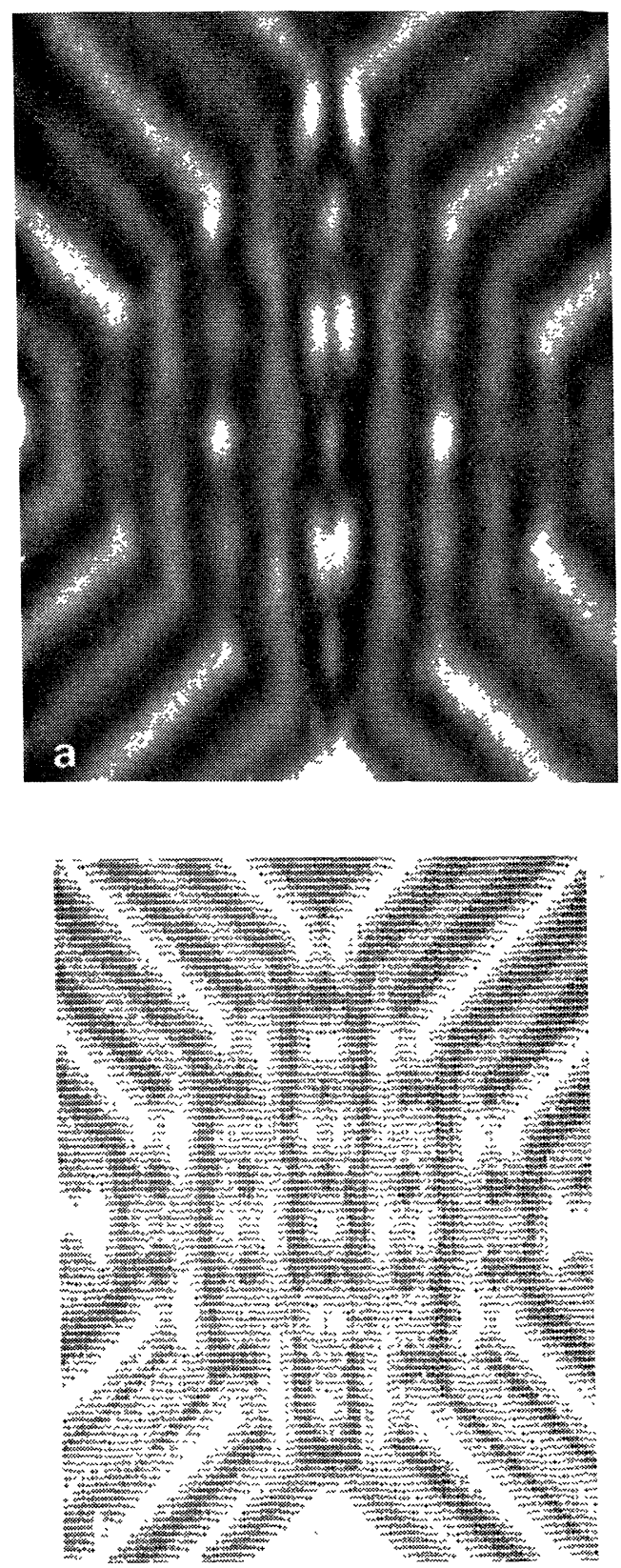

FIG. 6 a. - Micrographie $(1 \mathrm{MeV})$ de croisement de défauts d'empilement introduisant le même déphasage $\alpha=-\frac{2 \pi}{3}: w=0$.

b) Simulation correspondante.

Conclusion. - Les résultats obtenu smontrent l'intérêt des simulations de contrastes pour l'étude des défauts. Ces simulations peuvent être toutefois améliorées par deux procédés. Le premier consiste à imprimer les résultats à la sortie de l'ordinateur sur des surfaces plus étendues. Actuellement les simula- tions ont une taille de $33 \mathrm{~cm}$ de côté. La réduction plus importante donne à l'œil une sensation de variation d'intensité plus continue (il est possible également d'effectuer la sortie des résultats sur table traçante). Le second point consiste à améliorer l'échelle des noircissements que nous utilisons. Les résultats ci-dessus et ceux que nous avons obtenus récemment sur les simulations de dislocations montrent toutefois que notre méthode de simulation est suffisante pour l'étude détaillée de contrastes dus aux défauts.

\section{ANNEXE}

Construgtion. Franges D'Égale Épaisseur. - Si nous prenons des absorptions normale et anomale égales, soit $C_{0}=C_{g}$ et l'écart à l'angle de Bragg nul, le système de solution pourra s'écrire :

$$
\begin{aligned}
& \varphi_{0}=\frac{1}{2}\left\{\exp -i \pi z+\exp -\pi\left(2 C_{g}-i\right) z\right\}=\alpha+\beta \\
& \varphi_{g}=\frac{1}{2}\left\{\exp -i \pi z-\exp -\pi\left(2 C_{g}-i\right) z\right\}=\alpha-\beta .
\end{aligned}
$$

Pour écrire ces relations, nous utilisons les conditions aux limites :

$$
\varphi_{0}(0)=1 ; \quad \varphi_{g}(0)=0 .
$$

La construction phase-amplitude de $\varphi_{0}$ et $\varphi_{g}$ pour une épaisseur $z$ donnée consistera à appliquer la méthode de Fresnel (fig. 7).

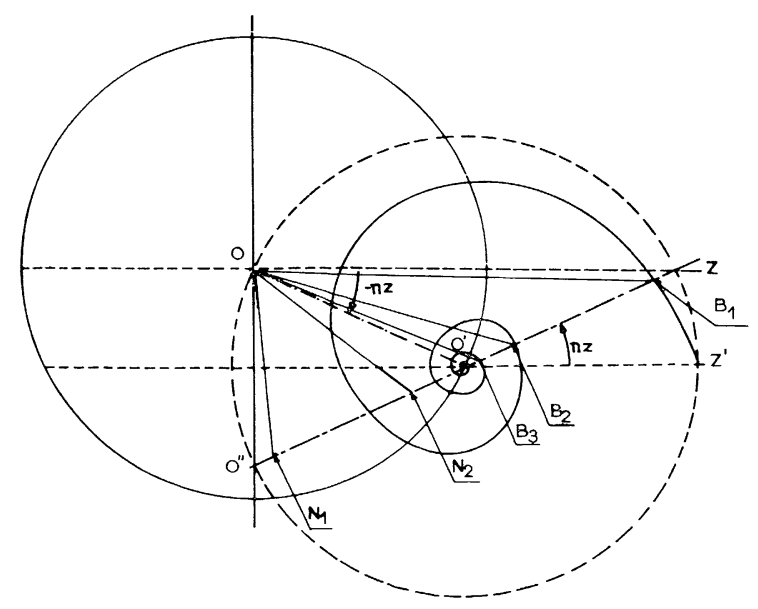

FIG. 7. - Construction des franges d'égale épaisseur.

L'extrémité $\mathrm{O}^{\prime}$ du vecteur $\boldsymbol{\alpha}$ décrira un cercle $C\left(\mathrm{O}, \frac{1}{2}\right)$. Le vecteur $\beta=\mathrm{O}^{\prime} \mathbf{B}$ de phase opposée à $\boldsymbol{\alpha}$ décrira une spirale logarithmique $S\left(\mathrm{O}^{\prime}, \frac{1}{2}\right)$.

Le point $\mathrm{B}$ extrémité de $\mathrm{OB}=\vartheta_{0} \quad$ se trouvera 
d'une part sur la spirale centrée sur l'extrémité $\mathrm{O}^{\prime}$ de $\alpha$ et sur l'axe d'angle de phase $+\pi z$. Cet axe est construit en joignant $\mathrm{O}^{\prime \prime} \mathrm{O}^{\prime}, \mathrm{O}^{\prime \prime}$ étant l'intersection de la perpendiculaire à l'axe $(\pi z=0)$, passant par $\mathrm{O}$, et du cercle $\left(\mathrm{O}^{\prime}, \mathrm{O}^{\prime} \mathrm{O}\right)$. L'onde transmise sera représentée par :

$$
\mathrm{OB}_{p}=\varphi_{\mathbf{0}}\left(\pi\left\{z+2(p-1) \xi_{g}\right\}\right)
$$

l'onde diffractée sera celle donnée par $O \mathbf{N}_{i}, N_{i}$ étant symétrique de $B_{i}$ par rapport à $\mathrm{O}^{\prime}$ :

$$
\mathrm{O} \mathbf{N}_{p}=\varphi_{g}\left(\pi\left\{z+2(p-1) \xi_{g}\right\}\right) .
$$

Cette construction met en évidence pour les grandes épaisseurs un fond continu de 0,25 I. Ce résultat n'est pas physique. Pour tourner cet écueil, il suffit de prendre l'absorption normale supérieure à l'absorption anomale. Les deux ondes sont alors absorbées. Le rôle de l'absorption normale étant le même pour les faisceaux transmis et diffractés, la modification à apporter sera d'introduire pour les ondes résultantes $\varphi_{0}$ et $\varphi_{g}$ un coefficient d'absorption supplémentaire $\varepsilon$ tel que :

$$
C_{0}=C_{g}+\varepsilon
$$

Remerciements. - Nous remercions M. le Directeur G. Dupouy des facilités obtenues dans l'utilisation du microscope de $1 \mathrm{MeV}$ à Toulouse.

\section{BIBLIOGRAPHIE}

[1] Rocher (A.) et Jouffrey (B.), Colloque de Lausanne de la SFME (1969).

[2] Rocher (A.) et Jouffrey (B.), Conference on NonConventional Electron Microscopy (1969), Oxford.

[3] Rocher (A.), Thèse de Spécialité, Orsay, 1969.

[4] Bethe (H.), Ann. Phys. Lpz., 1928, 87, 55.

[5] Jouffrey (B.) et Taupin (D.), Phil. Mag., 1967, 16, 703.

[6] Howie (A.) et Basinski (S. S.), Phil. Mag., 1968, 17, 1039.

[7] Moliere (K.), Ann. Phys. Lpz., 1939, 34, 461.

[8] Yoshioka (H.), J. Phys. Soc. Jap., 1957, 12, 628.
[9] Howie (A.) et Wheilan (M. J.), Proc. Roy. Soc., 1961, A 263, 217.

[10] Hirsch (P. B.), Howie (A.), Nicholson, Pashley et WHELAN (M. J.), Electron microscopy of thin crystals, London, Butterworths, 1965.

[11] Gevers (R.), ART (A.) et AMeinckx (S.), Phys. Stat. Sol., 1963, 3, 1563.

[12] Gevers (R.), ART (A.) et AMelinckx (S.), Phys. Stat. Sol., 1964, 7, 605.

[13] Hrad (A. K.), Aust. J. Phys., 1967, 20, 557.

[14] Jouffrey (B.), Daniei, (A.) et Escaig (B.), J. Physique, 1966, 27, C 3, 114.

[15] Ayrol_LES (R.), Thèse, Toulouse, 1968. 